\title{
Field Emission Properties of Vertically-Aligned Carbon Nanotubes Grown on Stainless Steel by Hot-Filament Chemical Vapor Deposition
}

\author{
Nguyen Khac Hiep ${ }^{1}$, Dang Nhat Minh ${ }^{2}$, Nguyen Thanh Hai ${ }^{3}$, Nguyen Dinh Dung ${ }^{1}$, Luong Nhu Hai ${ }^{1}$, \\ Phan Ngoc Hong ${ }^{1}$ and Nguyen Tuan Hong ${ }^{1, *}$ \\ ${ }^{1}$ Centre for High Technology Development, Vietnam Academy of Science and Technology (VAST), 18 Hoang Quoc Viet, \\ Hanoi 100000, Vietnam \\ ${ }^{2}$ ARC Training Centre in Surface Engineering for Advanced Materials, Faculty of Science, Engineering and Technology, Swinburne \\ University of Technology, PO Box 218, Hawthorn, VIC 3122, Australia \\ ${ }^{3}$ Advanced Materials Institute of BIN Convergence Technology (BK21Plus Global), Jeonbuk National University, Jeonju, \\ Jeonbuk, 54896, Republic of Korea
}

\begin{abstract}
Carbon nanotubes (CNTs) for field emission applications were grown directly on 304 stainless steel (SS304) by using hot-filament chemical vapour deposition (hot-filament CVD). Vertically-aligned CNTs (VACNTs) with 1.5-millimetre-diameter in shape were successfully grown on the SS304 substrate. We obtained efficient electron emission with turn-on fields as low as $0.66 \mathrm{~V} / \mu \mathrm{m}$, emitting currents of $1.5 \mathrm{~mA}$ at an applied field of about $1.5 \mathrm{~V} / \mu \mathrm{m}$. The VACNTs-SS304 emitter also demonstrated high stability in emission current of $860 \mu \mathrm{A}( \pm 12.5 \mu \mathrm{A})$ which even better than that of the VACNTs grown on conventional silicon. This VACNTs-stainless steel is a promising candidate for X-ray tubes that require small, bright, and cold emitters. [doi:10.2320/matertrans.MT-MN2019046]
\end{abstract}

(Received April 17, 2020; Accepted May 14, 2020; Published July 25, 2020)

Keywords: carbon nanotubes, hot-filament CVD, field electron emission, catalysis, stainless steel 304

\section{Introduction}

Owing to their extraordinary geometrical, electrical, and mechanical properties, carbon nanomaterials and particularly carbon nanotubes have been investigated as materials for diverse applications such as composites, energy storages and electron sources. ${ }^{1-6)}$ Among them, the field electron emission (FE) - direct assembly of the VACNTs on electrically conducting substrates is of great interest. The most obvious method to obtain such structures is the direct growth of carbon nanotubes on the conducting substrates by CVD methods. Due to its potential applications in semiconductor industries and the easy formation of catalytic particles for carbon nanotube production, VACNTs are conventionally grown on silicon ( $\mathrm{Si}$ ) substrate. However, $\mathrm{Si}$ is not the most suitable material for FE cathodes at which high mechanical robustness and good thermal conductivity are demandingly required. Moreover, minimizing the contact resistance between VACNTs and substrates is necessary to avoid the voltage drop that can saturate emitting current prematurely. Because of the underlying reasons, carbon nanotubes are favourably needed to be grown on metallic substrates for the FE devices.

In recent years, synthesis of the VACNTs on metallic substrates using CVD methods has been reported. ${ }^{7,8)}$ Using catalytic bilayer thin films, scientists were successful in producing the VACNTs on several bulk and foil metals such as copper, nickel alloys, stainless steel, titanium, aluminium. The underneath or the barrier layer such as aluminium, tantalum was used to be a barrier layer to assist efficient CNTs growth. Several modified CVDs have been utilized to produce carbon nanotubes such as floating-catalyst CVD, plasma-enhanced CVD and water-assisted CVD.

*Corresponding author, E-mail: nguyen.tuanhong@gmail.com
Recent studies have also shown interesting progress toward growth and application of high-quality carbon nanotubes on metals for field emission applications. ${ }^{3,4,9-11)}$ For example, S. Park et al. reported the direct synthesis of CNT emitter on metal alloy by Plasma enhanced CVD for X-ray generator. ${ }^{4)}$ I. Lahiri and co-worker investigated the multi-walled carbon nanotubes grown on pure copper substrate by thermal CVD. The low contact barrier and high conductivity of the $\mathrm{Cu}$ are attributed to the good electron emitters. ${ }^{11)}$ In particular, D. Li and colleagues reported the fabrication of an electron source based on CNTs-stainless steel cathode and used in the ionization source. ${ }^{12)}$ However, to our knowledge, there is no report on the VACNTs grown on stainless steel by hot-filament CVD and applied for field emission devices though they demonstrate good field emission properties.

In this paper, we presented the fabrication of the VACNTs on SS304 substrates for field emission applications. The hotfilament CVD involving decomposition of $\mathrm{CH}_{4}$ and $\mathrm{H}_{2}$ at a temperature range of approximately $2200-2500^{\circ} \mathrm{C}$ was developed to attain highly efficient growth of VACNTs on SS304. The hot-filament CVD facilitates the VACNT growth by high-temperature hydrocarbon disassociation without degrading catalyst efficiency. Field electron emission characteristics of the VACNTs-SS304 demonstrated low turn-on field, high and stable emitting current. Since Si had been widely used as a cathode material, our research includes the VACNTs grown on Si for comparing the field emission properties.

\section{Experimental Procedure}

\subsection{Materials preparation}

The VACNTs were produced by a three-step process consisting of the catalyst deposition and the pre-treatment, 
and the CVD process. The 304 stainless steel (SS304) substrate with a thickness of $500 \mu \mathrm{m}$ (item number: 753463, Nilaco, Japan) were used. The SS304 substrate was cleaned ultrasonically in acetone/isopropyl for $60 \mathrm{~min}$ each step before other experimental processes. To deposit catalytic thin films, the sequential sputtering of $\mathrm{Al} / \mathrm{Fe}$ in the Argon atmosphere $\left(2.5 \times 10^{-2}\right.$ Torr $)$ was employed. The nominal thickness of $\mathrm{Al}(40 \mathrm{~nm}), \mathrm{Fe}(5 \mathrm{~nm})$ were estimated from the pre-determined deposition rates. The catalysts are selectively deposited using a routine photolithography process. In the second step, substrates coated with such bilayers were loaded into a thermal furnace and heated in the ambient atmosphere. All the catalysts that were similarly annealed at temperatures ranging from 350 to $450^{\circ} \mathrm{C}$ resulted in the successful growth of CNTs. However, we found that the catalyst thin film pretreated at $400^{\circ} \mathrm{C}$ in 10 minutes is the best fit for carbon nanotube growth. In experiments and after the thermal treatment process, catalytic particles were characterized and they are homogenous and in nanosized (less than $30 \mathrm{~nm}$ in size, about $1.1 \times 10^{11}$ particles $/ \mathrm{cm}^{2}$ ).

The VACNT growth was carried out by using the hotfilament CVD method described elsewhere. ${ }^{13)}$ Methane (20 SCCM - standard cubic centimetre per minute) and hydrogen (30 SCCM) were used as the feedstock gas, 30-Torr pressure, and the growth time of fewer than ten minutes. A bias voltage on tungsten-made filaments (item number: F-205W, Nilaco, Japan) is gradually increased and the filament temperature was elevated to about $2200-2500^{\circ} \mathrm{C}$. Rapid insertion of the growing substrate (stainless, silicon) into the reactive zone (approximately $17 \mathrm{~mm}$ beneath the four-filament cartridge) is followed and hence the substrate is heated rapidly. The onset of carbon nanotube growth began at $600^{\circ} \mathrm{C}$; however, steady growth was obtained at about $700^{\circ} \mathrm{C}$. It is noted that hot-filament CVD has the edge on decomposing $\mathrm{CH}_{4} / \mathrm{H}_{2}$ in relatively high temperature that is favourable for the rapid growth of CNTs. ${ }^{13)}$

\subsection{Characterization preparation}

The as-produced VACNTs were examined by a fieldemission scanning electron microscope SEM (Hitachi FESEM S-4800) and Raman spectroscope (IFS/66 Bruker system). Carbon nanotubes were also analysed by transmission electron microscope TEM (JEOL-JEM3011, 300 $\mathrm{keV})$.

The 1.5-millimetre-diameter VACNT on both SS304 and highly doped silicon substrates are subject to field emission (FE) test to understand and compare their field emission properties. The FE measurement is carried out in a vacuum chamber (KVM-T4060 system, Korea Vacuum) with the working pressure of $5 \times 10^{-6}$ Torr and a ballast resistor of $200 \mathrm{~K} \Omega$. Keithley-248 (Tektronix, USA) is a DC anode voltage source. Emission electron current is measured by Keithley-2001 (Multi-meter, Tektronix, USA). FE data are acquired using LABVIEW software and a personal computer through a general-purpose interface bus interface (GPIB) card. The diode configuration is set up for FE measurement with an aluminium anode, the VACNTs-SS304 cathode. The cathode back-side is glued by silver paint. The anode and the cathode were electrically isolated by Kapton sheets (spacer) with a gap which is defined as anode-to-CNT top-surface distance. We also install a CCD camera to monitor real emitting sites.

The field emission measurements were repeated at least tens of times by ramping anode voltage up and down. The electric field was calculated to be the anode voltage divided by the gap. Current densities were relatively estimated from the net currents and the VACNT size. The turn-on field refers to an electric field necessary to extract 100 nano-ampere (nA) of emission current following the conventional definition. The field emitter was also tested in a continuous condition or a stability test. Before the stability test, an ageing process was carried out. The short-term stability of the VACNT field emitter was evaluated by monitoring emission current under constant anode voltage, $100 \%$ duty cycle.

\section{Results and Discussions}

\subsection{Characterization data}

High-resolution scanning electron microscope (SEM) was employed to examine the as-produced VACNTs on SS304 in details. Figure 1 shows SEM images of the optimized catalyst and as-grown VACNTs. The catalyst thin films after the pre-treatment in the ambient air $\left(400^{\circ} \mathrm{C}, 10\right.$ minutes $)$ is shown in Fig. 1(a). In experiments, the buffer layer (Al) and the SS304 substrate both impact on the catalyst morphology. Without the Al layer, the catalyst is of the large nanoparticles and low density. With the Al layer, the Fe/ $\mathrm{Al}(5 \mathrm{~nm} / 40 \mathrm{~nm}$ in thickness) thin films are strongly restructured and broken into nanosized particles after the thermal treatment. The catalytic nanoparticle is about $10-30 \mathrm{~nm}$ in diameter. The experiments also confirmed that the pre-treatment conditions had a strong effect on the nanoparticle density. Indeed, if the thermal annealing was less than $350^{\circ} \mathrm{C}$, the obtained catalyst was clustered rather than particle shaped. On the other hand, temperature annealing higher than $450^{\circ} \mathrm{C}$ results in catalyst particles in large size (heat-induced coalescence) and less dense.

Figure 1(b) and Fig. 1(c) show typical SEM images of the as-produced VACNTs (the aerial and the cross-section view) on SS304 substrate. The VACNTs, length ranges of 150 $200 \mu \mathrm{m}$, were achieved within the 10-minute CVD process. The VACNTs were homogeneous in the top surface, see Fig. 1(b). Observing high magnified image $\left(3 \times 10^{4}\right.$ times $)$, carbon nanotubes are about $10-30 \mathrm{~nm}$ in diameter, Fig. 1(c). This is good agreement with the catalyst size. It was found that the larger catalyst density, the higher carbon nanotube density which was necessary for the vertically-aligned carbon nanotube growth. The growth of carbon nanotubes on SS304 is experimentally terminated within ten minutes of hotfilament CVD process due to catalyst degradation. In fact, the VACNT thickness grown on $\mathrm{Si}$ are longer when compared with those on the SS304 most likely due to more unexpected diffusion of the $\mathrm{Al} / \mathrm{Fe}$ catalyst into the metal than the $\mathrm{Si}$ surface.

Figure 2 shows typical Raman spectra of the carbon nanotubes produced on $\mathrm{SS} 304$ and $\mathrm{Si}$ substrate for comparison. Raman signature was recorded at room temperature using an excitation laser wavelength of $1064 \mathrm{~nm}$. There is no apparent difference in the spectra peaks of two VACNTs. The main feature at $\sim 1606 \mathrm{~cm}^{-1}$, which accounts 

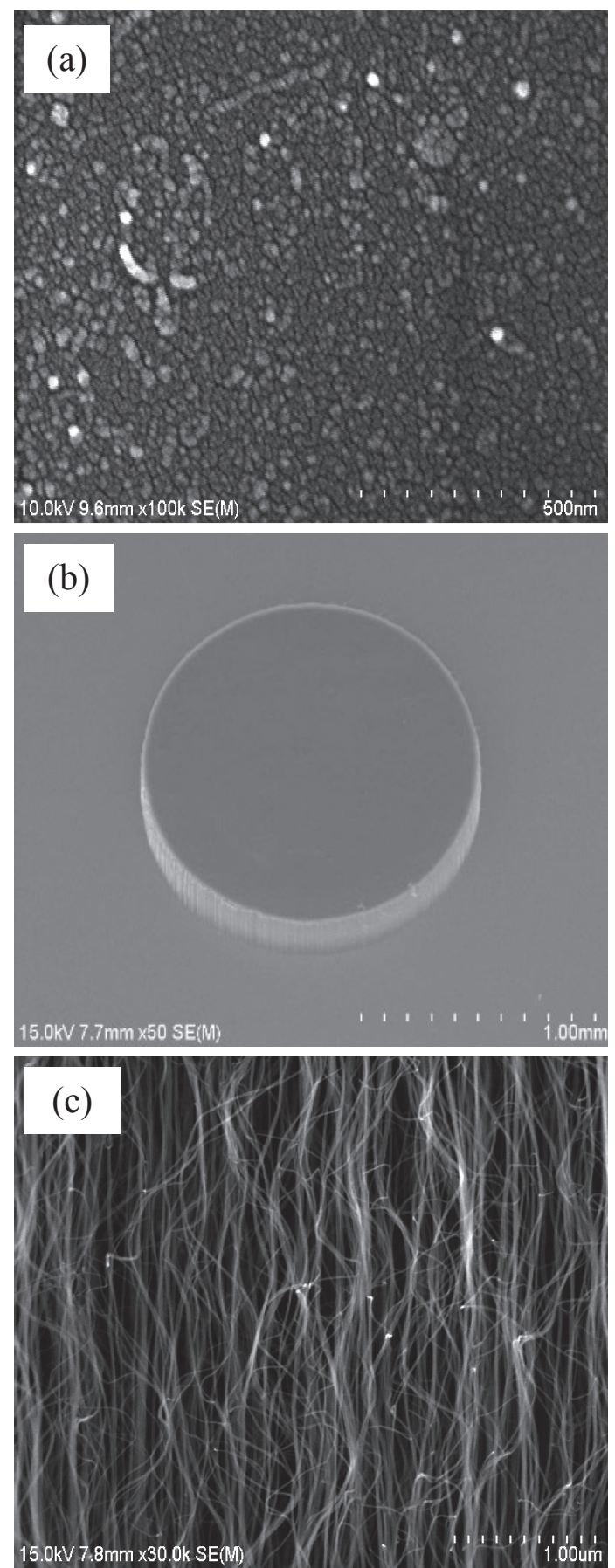

Fig. 1 (a) SEM images of $\mathrm{Fe}-5 \mathrm{~nm} / \mathrm{Al}-40 \mathrm{~nm}$ catalyst after thermal treatment in ambient air $\left(400^{\circ} \mathrm{C}, 10\right.$ minutes $)$ and VACNTs on SS304 substrate (b) aerial view; (c) cross-section view.

for the graphite G-peak frequency, is in good agreement with the typical Raman G-band frequency recorded for multi-walled CNTs. The greater disordered peak (D-band) at $\sim 1286 \mathrm{~cm}^{-1}$ implies that there is either some amount of amorphous carbon contaminants or defected nanotubes. Also, the $\mathrm{G}^{\prime}$-band mode, arising from the two-phonon process, was observed at $2534 \mathrm{~cm}^{-1}$. It is worth noting that the two-phonon process, such as the $\mathrm{G}^{\prime}$-band mode, would not be observed if the graphitic structure was too poor. The appearance of the $\mathrm{G}^{\prime}$-band mode implies that both VACNT samples were clean and that the content of carbonaceous impurities was insignificant. ${ }^{14)}$ Hence, it can be said that two

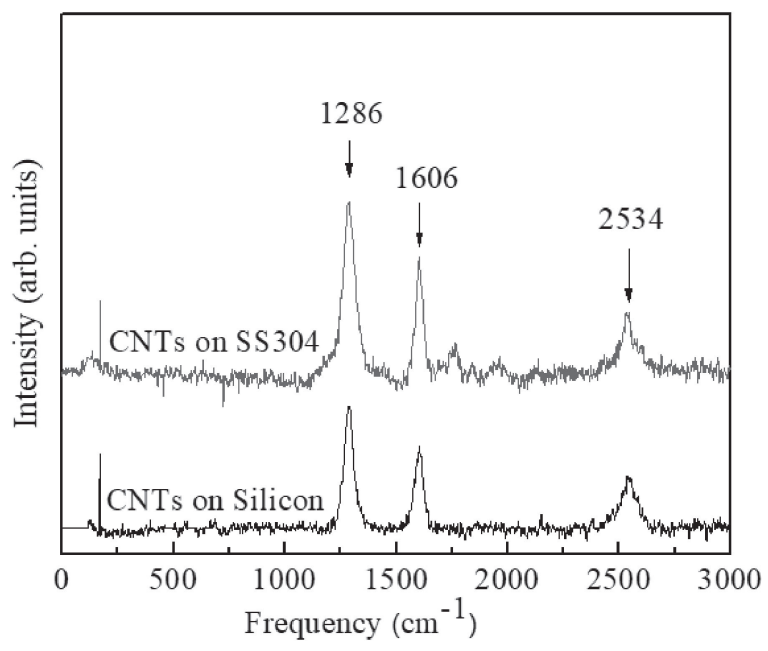

Fig. 2 Raman spectra of VACNTs produced on SS304 and silicon substrate.

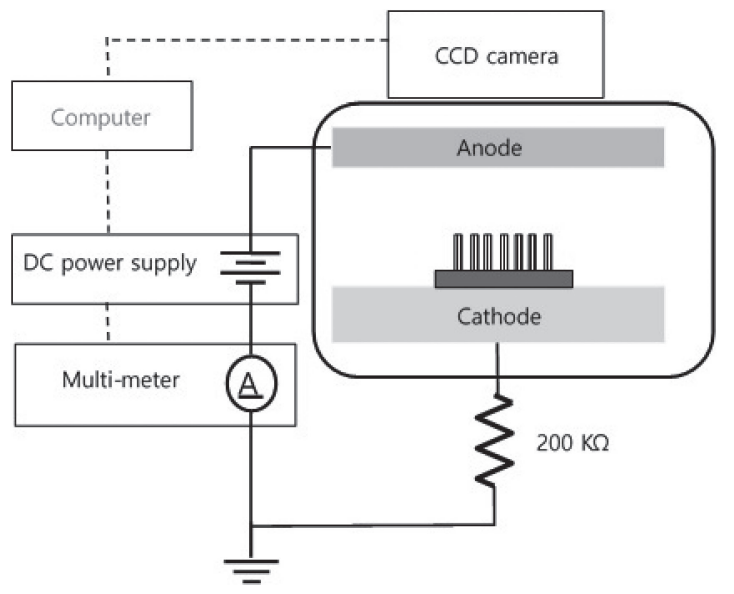

Fig. 3 Schematic diagram of field emission measuring (FEA) setup with Keithley-248 voltage supply, Keithley-2001 multi-meter, and ballast resistor of $200 \mathrm{k} \Omega$.

VACNTs are alike in properties whatever on SS304 or Si substrate.

\subsection{Field emission properties}

Field emission characteristics are carried out using the measuring setup shown in Fig. 3. The 1.5-millimetrediameter VACNTs with a thickness of about $150 \pm 20 \mu \mathrm{m}$ on both SS304 and the highly-doped silicon substrate are tested. The diode gap is approximately $1450 \mu \mathrm{m}$ for the $\mathrm{SS} 304$ and $1500 \mu \mathrm{m}$ for the Si. Figure 4(a) showed $I-V$ curves, net current (mA) versus applied anode voltage (V), of two VACNT cathodes. We simply estimate the area of the VACNTs as the emission area $\left(\Phi-1.5 \mathrm{~mm}\right.$ shape, $\left.1.76 \mathrm{~mm}^{2}\right)$. The turn-on field is defined as the corresponding field to extract $0.1-\mu \mathrm{A}$ emitting current. The $I-V$ curves were repeatedly measured several times before taking into characterization. The VACNT-SS304 cathode shows the turn-on field of $0.66 \mathrm{~V} / \mu \mathrm{m}$, higher than that of the $\mathrm{VACNT} / \mathrm{Si}(0.57 \mathrm{~V} / \mu \mathrm{m})$. Both two emitters demonstrate emitting currents of about $1.5 \mathrm{~mA}$ (about $85 \mathrm{~mA} / \mathrm{cm}^{2}$ ) at an applied field of $1.5 \mathrm{~V} / \mu \mathrm{m}$. Individually, CNTs are good 
(a)

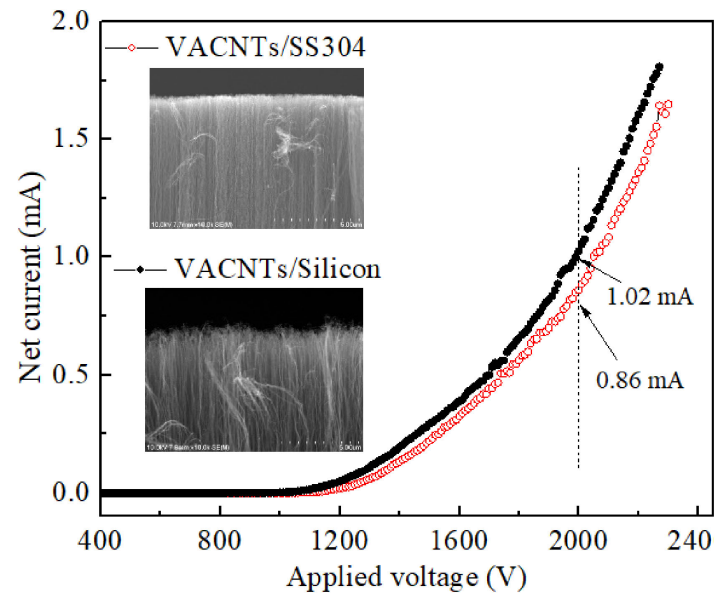

(b)

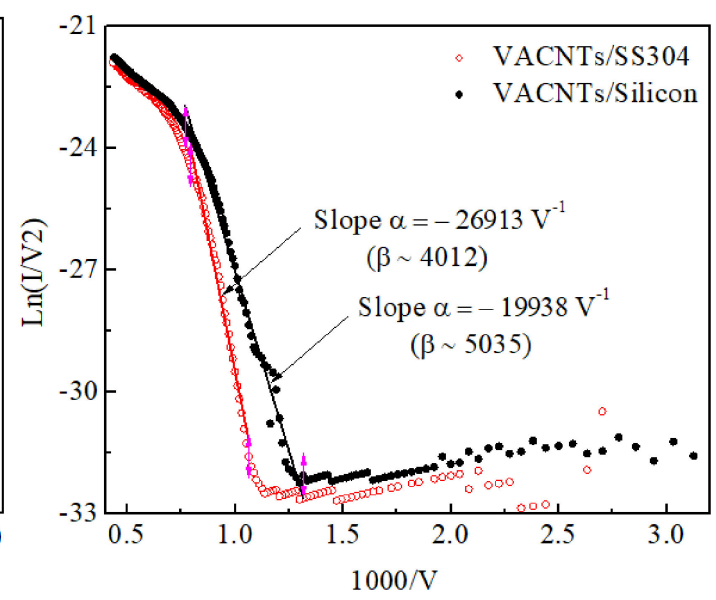

Fig. 4 (a) I-V curves of 1.5-mm.dia. VACNTs on SS304 and silicon substrate and inset SEM images of corresponding two CNTs top-surface; (b) Corresponding F-N plots.

emitters due to their high aspect ratio in geometry. However, for the VACNTs grown by CVD, nanotube density is high, and they are entangled each other. This results in the screen effect that reduced the field enhancement factor of carbon nanotubes. ${ }^{15)}$ To explain the above results, we performed SEM analysis of the VACNT top-surface morphology. It is worth noting that the $\mathrm{Si}$ substrate and $\mathrm{Fe} / \mathrm{Al}$ catalyst are excellent for VANCT synthesis by the hot-filament CVD, ${ }^{13}$ ) high growth rate (up to $100 \mu \mathrm{m}$ per minutes). The rapid growth of the VACNTs easily gives rise to nanotube bundles extruding on the top-surface. In contrast, the VACNTs-SS304 growth is more homogeneous and the as-grown VACNTs are flat, see inset in Fig. 4(a). Such extruded carbon nanotubes on the $\mathrm{Si}$ would mitigate the screen effect and hence, emit electron current at a lower applied field.

A Fowler-Nordheim (FN) plot, $\ln \left(\mathrm{I} / \mathrm{V}^{2}\right)$ versus $1 / \mathrm{V}$, is shown in Fig. 4(b). Generally, a linear F-N plot is attributed to a quantum tunnelling process through a potential barrier that is thinned because of the electric field. Theoretically, F-N equation is given in terms of the emission current $\mathrm{I}$ in ampere, emission area $\mathrm{A}$ in square-meter, applied bias voltage $\mathrm{V}$ in volt, cathode-anode gap d in meter, work function $\phi(5 \mathrm{eV})$ in $\mathrm{eV}$ as following:

$$
I=A \frac{1.42 \times 10^{-6}}{\phi} \beta^{2}\left(\frac{V}{d}\right)^{2} \exp \left(\frac{10.4}{\sqrt{\phi}}\right) \exp \left(-\frac{B \phi^{1.5} d}{\beta V}\right)
$$

where $\beta$ is the field enhancement factor, and $\mathrm{B}=6.44 \times$ $10^{9} \mathrm{VeV}^{-1.5} \mathrm{~m}^{-1}$ is a constant. Therefore, the plot of $\ln \left(\mathrm{I} / \mathrm{V}^{2}\right)$ with respect to $1 / \mathrm{V}$ becomes linear, and the slope is given by $-B \phi^{1.5} d / \beta$. Using the slope that was deduced by fitting the linear segment of the F-N plot to a straight line together with the known values for $\mathrm{d}$ and $\mathrm{B}$, we estimated the field enhancement factor $\beta$. We linearly fit the F-N plots which are illustrated in Fig. 4(b), and the obtained fit closely follows the measured FE characteristics up to the slow saturation (onset at $0.82 \mathrm{~V} / \mu \mathrm{m}$ for the SS304 and $0.77 \mathrm{~V} / \mu \mathrm{m}$ for the $\mathrm{Si})$. It confirmed that the measured emitting current follows the quantum tunnelling theory. The field enhancement factor of the VACNTs on the SS304 and the Si are 4012 and 5035, respectively. The higher field enhancement factor of the
CNT-silicon cathode (5035) is attributed to the microscopically extruding morphology of carbon nanotubes on the top. Those extruded CNTs as evidenced in inset of Fig. 4(a) would mitigate the crow effect, and hence, enhance the local electric field. The saturation behaviour in the F-N plots has been previously observed and ascribed to the convoluted origins of space charge, adsorbates, thermionic emission, emitter geometry. ${ }^{16,17)}$ Another possibility is that the saturation is due to the presence of electrical resistance in series with the CNT emitter, which can be a ballast resistor and a contact resistance at the nanotube-substrate contact. In that case, the voltage drop $(\mathrm{U}=\mathrm{I} \times \mathrm{R})$ across resistance will lead to a decrease of the effective anode voltage, and therefore to a flattening of the characteristics. The SS304 substrate is expected to have the least contact resistance with CNTs as these are predominantly metallic, thus offering a low substrate-CNT resistance.

The stability of field emission current is another important factor in applications. Before the stability test, an ageing process was carried out until $I-V$ curves are stable. The constant anode voltage ( $100 \%$ duty cycle) equivalent to an electric field of approximately $1.33 \mathrm{~V} / \mu \mathrm{m}$ (for both the SS304 and the Si) was applied. Figure 5 demonstrated temporal emitting current in more than one hour. For the VACNTs-SS304 emitter, the deviation of the observed emitting current with a maximum fluctuation is only $12.5 \mu \mathrm{A}(1.5 \%$ in total current) for $860 \mu \mathrm{A}$. This small fluctuation meets the requirements in many applications. Having high electrical and thermal conductivity, hence, the SS304 substrate was assumed to minimize Joule effect during emission process, which highly impacts on emission stability. In contrast, the current deviation of the VACNTs-silicon is $25 \mu \mathrm{A}(2.5 \%)$ for $1020 \mu \mathrm{A}$ current. This current deviation is even significant in a sense that the Si substrate has larger the series resistance, which can mitigate the current fluctuation.

\section{Conclusion}

We have reported the successful VACNTs growth on 304 stainless steel by using hot-filament CVD. The related FE 


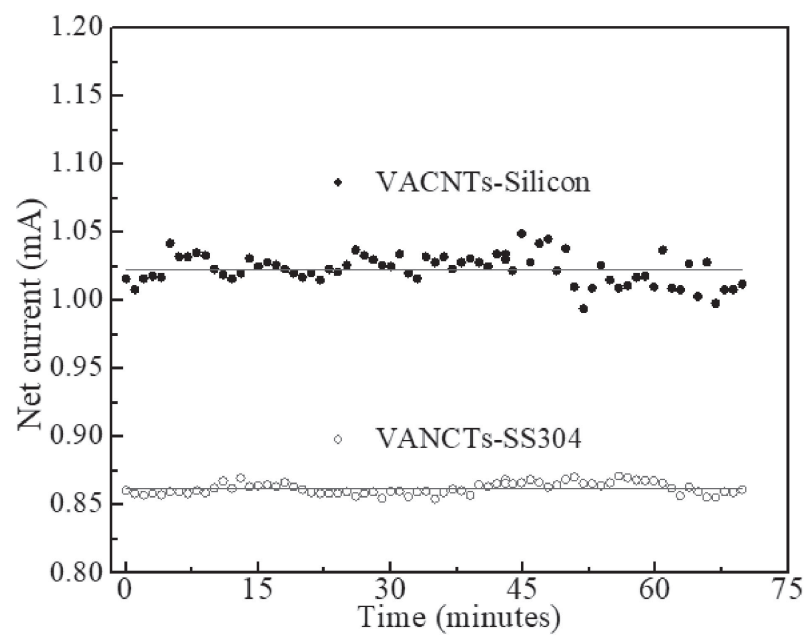

Fig. 5 Stability test of the VACNTs on SS304 and on Si, constant anode voltage of $2000 \mathrm{~V}(1.33 \mathrm{~V} / \mu \mathrm{m}), 100 \%$ duty cycle.

properties were tested. The 1.5-mm-dia. VACNTs-SS304 demonstrates the turn-on field of $0.66 \mathrm{~V} / \mu \mathrm{m}$; the cathode can reach emitting current of $1.5-\mathrm{mA}$ at $1.5 \mathrm{~V} / \mu \mathrm{m}$ field. These FE characteristics are not better than those of the VACNTs on the Si; however, the VACNTs-SS304 demonstrates good stability in emission current. This VACNTsstainless steel is a promising candidate for the miniature $\mathrm{X}$-ray tubes which require small, bright field cathodes.

\section{Acknowledgements}

This research is funded by Vietnam National Foundation for Science and Technology Development (NAFOSTED) under grant number 103.99-2016.58.

\section{REFERENCES}

1) H. Zein and Y.A. Al-Shataif: Mater. Trans. 61 (2020) 14-26.

2) D.N. Minh, H.P. Duong, L. Hoang, P.D. Nguyen, P.D. Tran and P.N Hong: Mater. Trans. 61 (2020) 1535-1539.

3) R. Longtin, J. Ramon Sanchez-Valencia, I. Shorubalko, R. Furrer, E Hack, H. Elsener, O. Gröning, P. Greenwood, N. Rupesinghe, K. Teo, C. Leinenbach and P. Gröning: Sci. Technol. Adv. Mater. 16 (2015) 015005.

4) S. Park, A.P. Gupta, S.J. Yeo, J. Jung, S.H. Paik, M. Mativenga, S.H. Kim, J.H. Shin, J.S. Ahn and J. Ryu: Nanomaterials 8 (2018) 378.

5) T. Noguchi, A. Magario, S. Fukazawa, S. Shimizu, J. Beppu and M. Seki: Mater. Trans. 45 (2004) 602-604.

6) M.N. Dang, T.D.T. Ung, H.N. Phan, Q.D. Truong, T.H. Bui, M.N Phan, L.Q. Nguyen and P.D. Tran: Mater. Lett. 194 (2017) 145-148.

7) X. Li, M. Baker-Fales, H. Almkhelfe, N.R. Gaede, T.S. Harris and P.B. Amama: Sci. Rep. 8 (2018) 4349.

8) M. Yilmaz, S. Raina, S.H. Hsu and W.P. Kang: Mater. Lett. 209 (2017) 376-378.

9) A.P. Gupta, S. Park, S.J. Yeo, J. Jung, C. Cho, S.H. Paik, H. Park, Y.C. Cho, S.H. Kim, J.H. Shin, J.S. Ahn and J. Ryu: Materials (Basel) 10 (2017) 878.

10) Y. Liu, X. Miao, J. Fang, X. Zhang, S. Chen, W. Li, W. Feng, Y. Chen, W. Wang and Y. Zhang: ACS Appl. Mater. Interfaces 8 (2016) 52515260 .

11) I. Lahiri, R. Seelaboyina, J.Y. Hwang, R. Banerjee and W. Choi: Carbon N.Y. 48 (2010) 1531-1538.

12) D. Li, Y. Cheng, Y. Wang, H. Zhang, C. Dong and D. Li: Appl. Surf Sci. 365 (2016) 10-18.

13) N.T. Hong, S.Y. Kim, K.H. Koh and S. Lee: Thin Solid Films 519 (2011) 4432-4436.

14) R.A. DiLeo, B.J. Landi and R.P. Raffaelle: J. Appl. Phys. 101 (2007) 064307.

15) L. Nilsson, O. Groening, C. Emmenegger, O. Kuettel, E. Schaller, L. Schlapbach, H. Kind, J.-M. Bonard and K. Kern: Appl. Phys. Lett. 76 (2000) 2071-2073

16) L. Chen, Z. Ji, Y. Mi, H. Ni and H. Zhao: Phys. Scr. 82 (2010) 035602.

17) L.-A. Gautier, V. Le Borgne, S. Al Moussalami and M. El Khakani: Nanoscale Res. Lett. 9 (2014) 55. 